\title{
Broad Emission Lines in Active Galactic Nuclei
}

\author{
J. A. Bald win ${ }^{1}$
}

Cerro Tololo Interamerican Observatory, Casilla 603, La Serena, Chile ${ }^{2}$

\begin{abstract}
This paper assesses what we have learned about the structure of AGN broad-line region (BLR), with emphasis on the work other than reverberation studies in order to complement other recent reviews. Basic photoionization models are briefly described. Current models of the BLR and some of the observations that might distinguish among them are discussed.
\end{abstract}

\section{Some Basic Facts ${ }^{3}$}

Broad emission lines with full widths at zero intensity of up to many thousands of kilometers per second are a characteristic feature of most types of AGN. These lines originate in the broad-line region (BLR). Some of the wide variety of models attempting to describe the BLR, and the observational evidence bearing on its structure, will be described below. It will be seen that, in spite of the great advances made over the past decade through reverberation mapping and other techniques, the typical structure of the BLR is still not very well defined.

What do seem to be reasonably well established are the following 'facts':

- Some appreciable part of the $B L R$ is photoionized. This is of course what happens when a bright source of ionizing photons is brought together with cool gas, but there is always the possibility that the gas is shielded from the AGN continuum source and that the excitation could be mechanical. Reverberation studies of several low-luminosity AGN (see Peterson 1994 and references therein) show a direct response of the emission-line strengths to changes in the continuum strength, firmly establishing that at least the variable component of the emission lines comes from photoionized gas.

- The BLR is stratified. This has become widely accepted on the basis of the sizes deduced from the reverberation response of different emission lines. It is also suggested by the differences between line profiles, particularly those of high- and low-ionization species (\$4.1.).

\footnotetext{
${ }^{1}$ Visiting Astronomer, Institute of Astronomy, Cambridge, UK.

${ }^{2} \mathrm{CTIO}$ is operated by AURA, Inc. under cooperative agreement with the National Science Foundation.

${ }^{3}$ Unless they turn out to be wrong
} 
- Some appreciable part of the BLR is optically thick. Early models described the BLR as an ensemble of identical clouds (cf. Davidson \& Netzer 1979; Kwan \& Krolik 1981) which had to be optically thick in the Lyman continuum in order to explain the presence of low-ionization lines such as $\mathrm{Mg}$ II 2800; this is still required of the clouds in the outer part of a stratified BLR. More directly, the fact that lines such as Mg.II, Ly $\alpha$, and the Balmer lines respond to continuum changes shows that the variable components of these lines originate in optically thick gas. However, gas which is optically thin in the Lyman continuum can still produce the reverberation response of higher-ionization lines such as C IV. There are many lines of evidence that optically thin gas is present, probably mixed in with the optically thick gas (Shields et al. 1995).

- The BLR gas intercepts 5-25\% of the continuum light. This is deduced from the equivalent width of Ly $\alpha$ combined with simple photoionization models, assuming that the continuum source emits isotropically on the size scale out to the BLR (obscuring tori, etc., farther out could still make the continuum source appear to us to be anisotropic). The continuum source in most cases cannot be fully covered by optically thick gas, because the Lyman continuum edge usually is not seen in absorption (Smith et al. 1981; Koratkar et al. 1992).

Many of the preceding arguments draw on results from reverberation studies. These have been carried out for only a modest number of low-luminosity objects. It is not clear that all inferences from reverberation studies will extend to QSOs which are 100 or more times more luminous. The balance of radiative and gravitational effects may be very different in such objects. However, the pseudo-facts listed above seem likely to be correct in spite of such caveats.

\section{Modeling Individual BLR 'Clouds'}

Photoionization models of the BLR gas are usually described in terms of the properties of 'clouds', but it is implicit that this gas may in fact be arranged in anything from spherical clouds (either large or small compared to their ionized depths), to thin sheets, to the extended atmospheres, winds or shock-compressed regions surrounding stars, to the surfaces of accretion disks. Photoionization models just describe what happens as a function of depth behind an illuminated face, and predict the total emitted spectrum, irrespective of the 'cloud' context.

There are a number of similar photoionization codes; the one in widest public use is CLOUDY ${ }^{4}$ (Ferland 1996). The input parameters for any of these codes include the ionizing continuum flux and the gas density at the front face. These often are combined into a single ionization parameter, $U=\Phi / c n_{\mathrm{H}}$ where $\Phi$ is the flux of ionizing photons and $n_{\mathrm{H}}$ is the hydrogen gas density (Netzer 1991). Besides this, it is necessary to specify at least the continuum shape, the dependence of density or pressure on depth, the column density $N(\mathrm{H})$, and the abundances.

\footnotetext{
${ }^{4} \mathrm{~A}$ copy of this code can be obtained at http://www.pa.uky.edu/ gary/cloudy.
} 
The most important computational limit of these models is their approximate treatment of radiative transfer, usually through the escape probability technique (cf. Avrett \& Loeser 1988; Collin-Souffrin \& Dumont 1989). Escape probabilities are not expected to predict upper-level populations accurately, but should do a good job with line thermalization. Probably the weakest predictions are the hydrogen Balmer and higher series at high densities. The answer will await a definitive calculation.

However, there are also a host of problems of interpretation, where the models give essentially the correct answers but it is unclear what the input parameters should be. These include:

What input spectrum does the BLR see? The predicted emission-line spectrum is sensitive to the exact shape of the 'blue bump' in the UV and soft X-ray continuum, since it is photons at these energies that govern the ionization level and excitation of most of the observed emission lines (cf. Krolik \& Kallman 1988; Binette et al. 1989). In addition, free-free heating should be important at the high flux densities in the BLR, causing intensity ratios such as C IV/Ly $\alpha$ and $\mathrm{OVI} / \mathrm{Ly} \alpha$ to depend strongly on the position of the $0.1-1 \mathrm{~mm}$ low-frequency continuum cutoff (see Fig 2 of Ferland et al. 1992). Not only does the observed shape of the spectrum in these key regions change from object to object, correlating with both luminosity and radio power (cf. Mushotzky et al. 1993; Elvis et al. 1994), but the exact spectrum that would be seen by the BLR depends on the geometry of the BLR and continuum source and is therefore model-dependent.

Profile asymmetries. The emission lines in which the clouds are optically thick will preferentially escape from the illuminated face. This will make the line profiles more asymmetric than is observed if the gas motions are dominated by outflow or infall (Wilkes \& Carswell 1982; Laor et al. 1995).

Turbulence. The gas inside the individual clouds is usually assumed, without justification, to have no turbulent velocity. Large turbulent velocities will greatly decrease the optical depths in resonance lines, with a big effect on the strengths and possible asymmetries of these lines.

Cloud confinement. It does not appear possible to confine isolated clouds strictly through the pressure equilibrium of a two-phase medium (Mathews \& Ferland 1987). The clouds may be confined magnetically (Rees 1987), temporarily by shock fronts (Perry \& Dyson 1985), or may just expand and dissipate. In any of these situations, clouds with a wide range of densities can exist side by side.

There are also some longstanding problems or difficulties which now appear to be solved:

It is hard to explain the whole emission-line spectrum with one cloud. This has manifested itself both through the $\mathrm{Ly} \alpha / \mathrm{H} \beta$ intensity ratio being far lower than the Case B predictions (Baldwin 1977; Davidsen et al. 1977) and through the low-ionization lines being observed to carry away much more energy relative to the high-ionization lines than was predicted (Collin-Souffrin 1986). The explanation almost certainly lies in the stratification of the BLR, with the different lines being produced in different gas clouds (but see Collin-Souffrin et al. 1997).

The Fe II strength. Fe II is observed to be a major coolant, yet its strength has long been underpredicted by a large factor (Wills et al. 1985). However, new calculations (Verner et al. 1995 and in preparation) using a more complete 
model of the iron atom and new atomic constants appear to predict an FeII emission strength in much better agreement with the observations.

Measuring abundances. It has been difficult to use BLR spectra to measure the relative abundances of the elements, because the intensities of the strong lines reflect either the heating or the cooling rate, which are always in balance. However, intensity ratios like $\mathrm{N} v \lambda 1240 / \mathrm{C}$ IV $\lambda 1549$ and $\mathrm{N} v \lambda 1240 / \mathrm{He}$ II $\lambda 1640$ give the overall metal abundance $Z$, under the assumption that nitrogen is built up as $Z^{2}$ in stellar enrichment processes (Hamann \& Ferland 1993; Ferland et al. 1996).

\section{Models of BLR Structure}

\subsection{Locally Optimally Emitting Clouds}

Several models have been developed describing the BLR in terms of parameterized cloud distributions, with no pretense at explaining how the gas came to be distributed in that manner. Rees et al. (1989) calculated the integrated emission-line spectra expected for a variety of one-to-one relations between radius, cloud density, and covering factor. Goad et al. (1993) and O'Brien et al. $(1994,1995)$ computed the reverberation response functions for similar models.

A recent extension of this idea is to suppose that the BLR consists of clouds spread over a wide range in distance $r$ from the ionizing source and with a wide range in gas density $n_{\mathrm{H}}$ at each $r$. Clouds will only emit strongly in a particular line if they have the correct combination of ionization parameter and density to efficiently form that line. Since the ionization parameter $U$ depends on both $r$ (through the ionizing photon flux $\Phi$ ) and $n_{\mathrm{H}}$, a given emission line is formed efficiently only in a specific area on the $r-n_{\mathrm{H}}$ plane. This area of optimum efficiency is different for different lines. This just maps out quantitatively the obvious point that if the BLR is a mix of all sorts of clouds, higher-ionization lines will be formed at smaller $r$. The predicted integrated BLR spectrum is then calculated by integrating over the full distribution function of clouds at all radii and densities. This is called the 'locally optimally emitting cloud' (LOC) model (Baldwin et al. 1995; Korista et al. 1996).

An important result from the LOC model is that once there are clouds with a wide distribution of properties scattered over a wide range of radii, the predicted integrated spectrum has only weak sensitivity to many of the parameters of the cloud distribution over much of their range, and is quite a reasonable match to the average observed spectrum. This is illustrated in Figs. 1 and 2. The integrated spectrum depends only weakly on the shape of the ionizing spectrum, on the column density of the clouds (and therefore presumably also on the distribution of column densities), and on the cloud distribution with $r$ (here parameterized as $\propto r^{\gamma}$ ) for $\gamma>-1$. This implies that in typical cases the BLR spectrum is determined more by powerful selection effects than by details of the cloud properties.

The parameters to which the LOC models do seem to be sensitive are the abundances and the distribution of cloud densities. The abundance effect appears in Fig. 1 as the factor 3-10 underprediction of the strength of $\mathrm{N} v$ for the solar abundances used here. The overabundance of $N$ required to fit the LOC models is the same as has been found using single-zone models (Hamann 


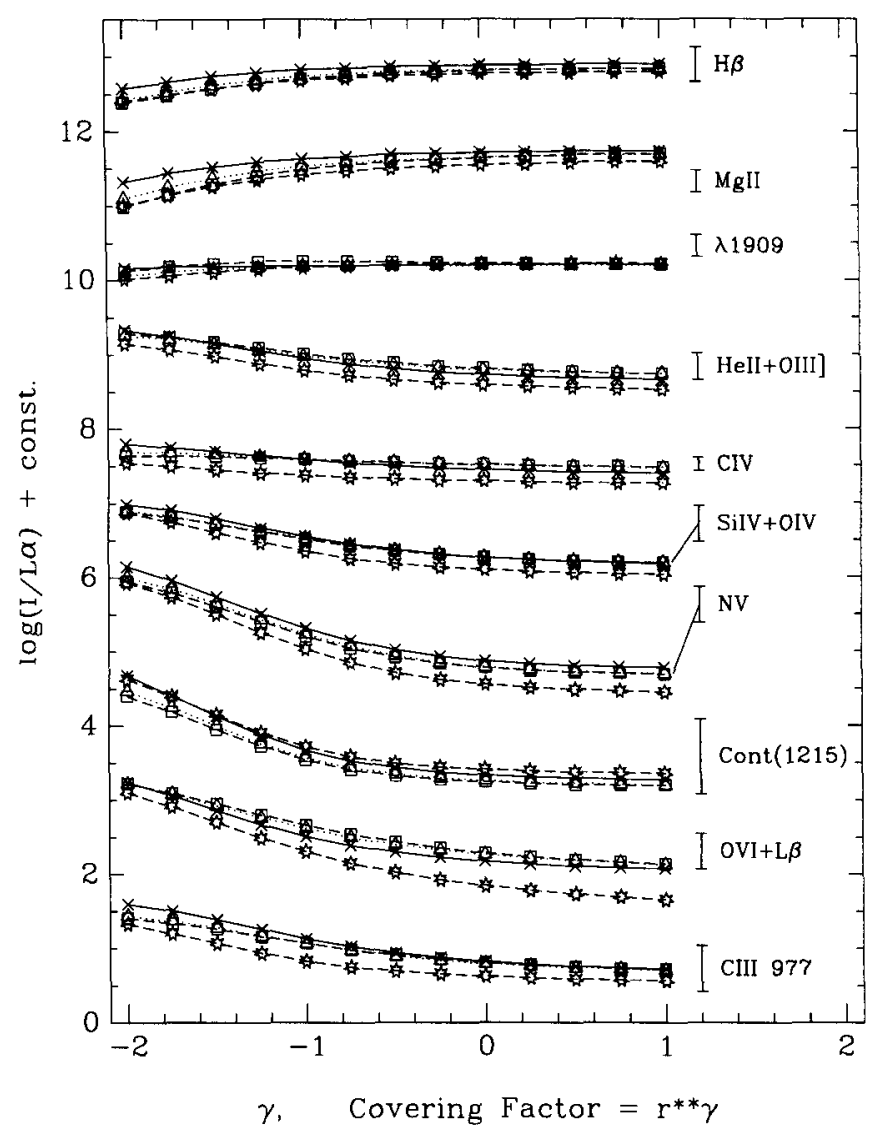

Figure 1. The curves show predicted intensity ratios relative to Ly $\alpha$ for the most important emission lines and for the continuum at $1215 \AA$, illustrating the relative insensitivity of the LOC models to most input parameters. These can be compared to the measured values indicated by the error bars at the right end of each set of curves; the values for the nine emission lines represent the range among the various composite spectra listed in Table 1 of Baldwin et al. (1995), while the values for the $1215 \AA$ continuum are for the typically observed $100 \AA$ equivalent width assuming $100 \%$ (top of error bar) through $10 \%$ (bottom of error bar) cumulative covering factor. The abscissa is the power-law exponent of the dependence on radius of the covering factors of the individual clouds; $\gamma=0$ implies a uniform distribution of clouds in space, while smaller $\gamma$ represents cloud distributions concentrated more towards the center. The symbols on the curves are: $x=$ hard continuum (44 eV peak for Blue Bump), $N(\mathrm{H})=10^{22} \mathrm{~cm}^{-2} ; \Delta=$ hard continuum, $N(\mathrm{H})=10^{23} \mathrm{~cm}^{-2} ; \square=$ hard continuum, $N(\mathrm{H})=10^{24} \mathrm{~cm}^{-2} ; \star=$ soft continuum (22 eV peak for Blue Bump), $N(\mathrm{H})=10^{23} \mathrm{~cm}^{-2}$. 


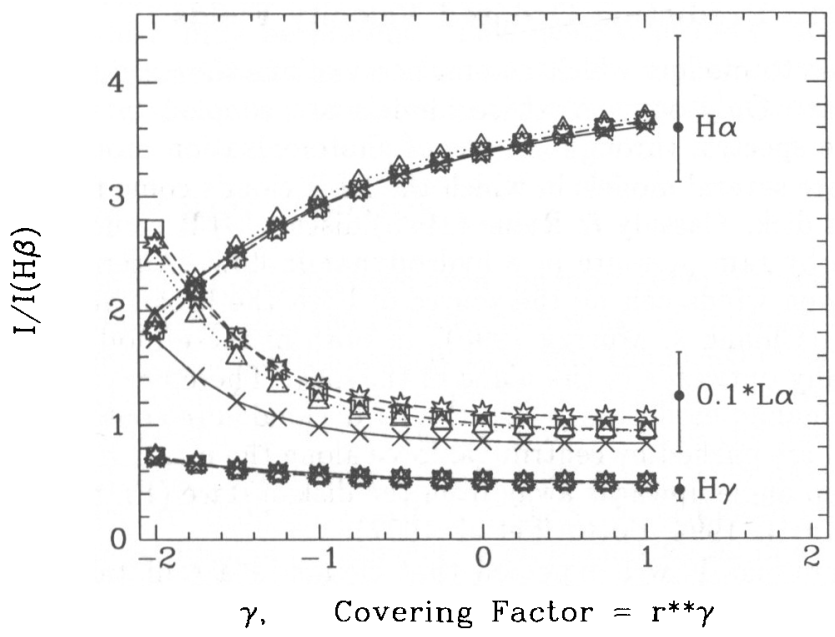

Figure 2. Hydrogen line intensity ratios for LOC models, compared to the average and range of measured values as given by Collin-Souffrin et al. (1997).

\& Ferland 1993). To date only simple power-law forms of the distribution of cloud densities have been tried; good matches to the observations are obtained with distribution functions $N\left(n_{\mathrm{H}}\right) \propto n_{\mathrm{H}}^{-1}$, where $N\left(n_{\mathrm{H}}\right)$ is the number of clouds of gas density $n_{\mathrm{H}}$. After correctly adjusting the two parameters which set the metal abundance and cloud-density distribution, the LOC models are expected to give a reasonable fit to all of the strong BLR lines, including the hydrogen spectrum (Ly $\alpha: \mathrm{H} \alpha: \mathrm{H} \beta: \mathrm{H} \gamma$ ).

The power-law dependences on $r$ and $n_{\mathrm{H}}$ have been used to test the sensitivity to a broad distribution function. The actual coverage of the $r-n_{\mathrm{H}}$ plane, especially in an individual BLR, may be very spotty; a good example is the QSO 0207-398 described below. To the extent that they can be used to determine how much of the $r-n_{\mathrm{H}}$ plane is populated, and what parts are not populated, the LOC models can be an important tool for deducing BLR structure.

\subsection{Dust-Bounded BLR}

An important finding is that the radius of the BLR coincides at least roughly with the radius at which dust should no longer be evaporated by the radiation field of the central source. This is indicated both by energetics (Barvainis 1987; Sanders et al. 1989) and by reverberation measurements (Clavel et al. 1989; Glass 1992). This leads to the concept that the onset of absorption by dust embedded in the emitting gas causes a drop in emissivity which marks the outer boundary of the BLR (Netzer \& Laor 1993). With a suitable velocity and density field, this can provide a natural break between the BLR and the narrow-line region, explaining the absence of broad forbidden lines. 


\subsection{Models Predicting Ordered Velocity Fields}

We turn now to models which attempt to explain the origin and dynamics of the BLR gas. Only some of these models are coupled to predictions of the emission-line spectra, through the use of photoionization models.

There are several models in which the BLR clouds come from the surface of an accretion disk. Cassidy \& Raine (1996) discuss BLR clouds stripped off the disk surface by ram pressure in a hydrodynamic flow. Alternatively, radiation pressure driven winds can be the source of both the broad absorption line and BLR clouds (Chiang \& Murray 1996). In both of these models the winds flow almost radially outwards in the plane of the disk. There are also a class of magnetohydrodynamic models in which the BLR clouds are contained by magnetic pressure and are pushed by centrifugal force along the magnetic field lines, which rise at a steep angle upwards away from the disk surface (Emmering et al. 1992; Königle \& Kartje 1994; Bottorff et al. 1997).

Ten years ago, it was expected that clouds in a radiation-pressure-driven wind would be disrupted because their optically thin edges would be accelerated much more efficiently than their very optically thick centers (Mathews 1986); however, the clouds in a stratified model such as in the LOC picture have lower column densities and undergo a much more uniform acceleration. These models therefore deserve to be revisited in general.

Another model involves the tidal disruption of stars within a few gravitational radii of the central black hole. Although some of the debris would be bound and maintain the random motions of the disrupted stars, they would emit principally continuum rather than line radiation. Roos (1992) has developed a scenario where the unbound portions of the disrupted star stretch into long radial tubes as they fly outwards, temporarily fragmenting into dense blobs which produce the BLR emission. The kinematics are thus similar to an outflowing wind.

Or it is possible that the high-ionization and low-ionization lines come from completely different structures; there are many observational indications of clearcut kinematic differences between the two types of gas (\$4.1.). One model for this is to have the low-ionization lines arise in gas on the surface of a rotating accretion disk, while the high-ionization lines come from gas being driven outward along the rotation axis of the disk (Collin-Souffrin et al. 1988).

\subsection{Models Predicting Random Velocity Fields}

'Bloated star' models associate the BLR gas with the extended atmospheres or stellar winds of evolved stars in a massive cluster surrounding the active nucleus (Scoville \& Norman 1988; Alexander \& Netzer 1994). Provided that these atmospheres are less diffuse than those of normal giants or supergiants, they are expected to avoid destruction in cloud-cloud (star-star) collisions in spite of the random velocity field. Using stars as a reservoir for BLR gas which is constantly dissipating also avoids problems with how to confine the clouds.

In the 'bouncing cloud' model (Mathews 1993), BLR clouds of unspecified origin can oscillate radially around stable orbits where radiation pressure and the gravitational attraction of the nucleus are in balance.

Perry \& Dyson (1985) proposed that the BLR clouds are temporary highdensity condensations behind the shock fronts which will inevitably be created 
by the supersonic motion of stars or supernova remnants through any hot, lowdensity medium which may be present. This general process should occur in most of the models mentioned above, and may be an important source of BLR clouds in addition to the ones postulated in the other specific models.

\section{Observations of BLR Structure}

Do the observations distinguish between even the most general classes of models? Reverberation results show that the red and blue wings of emission lines such as $\mathrm{H} \beta$ and C IV generally vary simultaneously to within the measurement errors (cf. Korista et al. 1995). At first sight this would seem to rule out systematic inflows or outflows as a major contributer to the variable parts of the line profiles; for example the recent HST reverberation results for NGC 5548 have been interpreted as a spherical distribution of clouds with either random circular orbits which take the clouds through a biconical beam of continuum light (Wanders \& Peterson 1996), or with slight inflow superimposed on nearly random motions (Done \& Krolik 1996). However, both the radiatively driven wind model (Chiang \& Murray 1996) and the MHD wind model (Bottorff et al. 1997) have also been shown to generally reproduce the NGC 5548 profile variability results, in spite of having highly ordered velocity fields. The reverberation results are thus ambiguous concerning the kinematic structure.

The remainder of this section describes what has been learned about the structure of the BLR from other observational approaches, and generally for very much more luminous objects where the ratio of radiative to gravitational forces may be much higher. This is intended to complement the extensive discussion of reverberation results presented elsewhere in this volume and in other recent reviews and conference proceedings (Peterson 1993; Gondhalekar et al. 1994).

\subsection{Can Different Cloud Structures be Identified?}

The question here is to what extent the profiles or strengths of different emission lines in individual AGN show that there is more than one kinematically distinct BLR component.

High-ionization vs. low-ionization lines. An important result is the general tendency for high-ionization lines to be blue-shifted relative to low-ionization ones and relative to the systemic velocity (Gaskell 1982; Wilkes 1986; Corbin 1990). Espey et al. (1989; see also Carswell et al. 1991) first extended this work to studies of individual objects over the full Ly $\alpha$ to $\mathrm{H} \alpha$ wavelength range by combining optical and infrared data. Similar studies combining ultraviolet and optical spectra, but comparing fewer different lines, have been carried out by Corbin (1991) and recently by Sulentic et al. (1995), Marziani et al. (1996) and Corbin \& Boroson (1996).

These studies show that in sample after sample some objects have large shifts between the peaks of different lines (up to a few thousand $\mathrm{km} \mathrm{s}^{-1}$ ) while other objects $(\sim 30-40 \%)$ have only small or undetectable shifts. The blueshifts of different lines are correlated, but different from line to line (cf. Corbin 1990). For a large sample, Tytler \& Fan (1992) find mean blueshifts of 0-400 $\mathrm{km} \mathrm{s}^{-1}$ for the different lines, with a tendency for the highest-ionization lines (e.g., 
He II $\lambda 1640$ ) to have the largest blueshifts (see their Fig. 9). Correlated with the shifts in the line peaks are progressively broader profiles, with asymmetric tails in the same direction as the shift of the line peak (Brotherton et al. 1994b; Marziani et al. 1996).

Do these kinematic differences mean that high- and low-ionization lines are formed in distinctly different structures, for example as in the Collin-Souffrin et al. (1988) model? Or does the rough sequence of increasing blueshift as a function of ionization level indicate a continuity between zones of different ionization, as in a virialized BLR with obscuration (or anisotropic emission from the clouds) blocking our view of the far (near) side? Several additional results seem to support the latter interpretation: the widths of the high and low ionization lines are at least roughly correlated, but with higher ionization lines having larger widths (Corbin 1991; Corbin \& Boroson 1996); the high/low ionization velocity difference becomes larger rather than smaller with increasing line width (Brotherton et al. 1994b); and at least in radio-loud objects the intensity ratio of the red wing of $\mathrm{Ly} \alpha / \mathrm{H} \beta$ decreases with increasingly redder continuum slopes in a way which is consistent with reddening (Netzer et al. 1995).

However, there are also results supporting a two-component model. Sulentic et al. (1995) find a complete lack of correlation between the $\mathrm{C}$ IV and $\mathrm{H} \beta$ velocity shifts (this is discussed further in §4.3.). Laor et al. (1995) find for a sample of low-redshift QSOs observed with HST that OVI $\lambda 1035$ is the same width as Ly $\alpha$, but at close to the systemic velocity as compared to significant blueshifts for lines like Ly $\alpha, \mathrm{C}$ IV and He II.

The intermediate-line and very broad-line regions. Many of the preceding results indicate a progressive range of properties among different emission lines. This can be described in terms of the linear combination of two different emission-line components (Brotherton et al. 1994a). These are an 'intermediateline region' (ILR) with narrow ( $2000 \mathrm{~km} \mathrm{~s}^{-1} \mathrm{FWHM}$ ), symmetrical profiles centered at the systemic velocity, and a 'very broad-line region' (VBLR) with broad $\left(\sim 7000 \mathrm{~km} \mathrm{~s}^{-1}\right)$ symmetrical profiles centered about $1000 \mathrm{~km} \mathrm{~s}^{-1}$ bluewards of the systemic velocity. Different lines include different proportions of the ILR and VBLR profiles; this can produce the full range of observed asymmetries and peak shifts in the different UV emission lines. This result was first obtained with a novel decomposition of the spectra of several hundred QSOs into 'principal components' which themselves have the form of spectra (Francis et al. 1992). It was then independently obtained by comparing composite spectra of broad-lined and narrow-lined QSOs (Wills et al. 1993; Brotherton et al. 1994b).

If the ILR and VBLR are taken to be physically distinct regions of the BLR, then fitting single-zone photoionization models to their average spectra indicates that the ILR lies at a radius of $\sim 1 \mathrm{pc}$ (for a luminous QSO) and has gas density $n_{\mathrm{H}} \approx 10^{10} \mathrm{~cm}^{-3}$, while the VBLR lies at about 10 times smaller radius and has a density $n_{\mathrm{H}} \approx 10^{12.5} \mathrm{~cm}^{-3}$. The ILR might be interpreted as a region bridging the gap between the VBLR and the narrow-line region (NLR).

However, it is unclear whether these results really imply physically distinct regions, or whether the ILR and VBLR should be considered as just the first two terms in some Fourier-like series describing the BLR spectrum. The only cases where a distinct inflection can be seen in the observed profiles, suggesting that 
they really are the superposition of two distinct shapes, are the very narrowestline QSOs.

Q0207-398: a case study. This luminous, $z=2.8$ QSO has at least three separate components in its UV emission lines (Baldwin et al. 1996). The narrowest component has a width of $\sim 1000 \mathrm{~km} \mathrm{~s}^{-1}$ (FWHM), and emits most strongly in $\mathrm{Al}$ III $\lambda 1860, \mathrm{Fe} I I \mathrm{l}$ and Fe II lines. This seems to come from gas which is so dense that the lines are thermalized, indicating $n_{\mathrm{H}} \approx 10^{12.7} \mathrm{~cm}^{-3}$. The derived distance from the continuum source is $r \approx 0.1 \mathrm{pc}$.

A second component has profiles which arise suddenly at the central velocity of the first component and have broad blueward tails, with FWZI $\approx 9000 \mathrm{~km} \mathrm{~s}^{-1}$. This gas emits strongly in C III $\lambda 977, \mathrm{OVI}, \mathrm{NV}$, and $\mathrm{CIV}$, and appears to be optically thin. It has $n_{\mathrm{H}} \approx 10^{11} \mathrm{~cm}^{-3}$, and is also at $r \approx 0.1 \mathrm{pc}$. One interpretation is to identify the first component with the extended atmospheres of stars and the second component with winds being stripped off the first component by radiation pressure; photoionization models show that radiation pressure could remove the atmospheres of even main sequence stars under the derived conditions.

The third and final component has symmetrical profiles with FWHM $\approx$ $1900 \mathrm{~km} \mathrm{~s}^{-1}$, centered at the same velocity as the first component. It is seen in Ly $\alpha, \mathrm{N} v, \mathrm{C}$ IV and $\mathrm{CHI}] \lambda 1909$. These are very similar to the parameters of the ILR discussed above. If this third component is indeed an ILR, it suggests that the first and second components might together represent the VBLR. However, this does not particularly imply that outflowing gas is a common feature of the VBLR (or BLR) of all high-luminosity AGN, because Q0207-398 has a very unusual spectrum in terms of the narrowness of the lines cores, the obviously different components in the profiles, and the presence of unusual lines.

\subsection{Orientation Effects}

Unified models predict that orientation effects are present in all AGN spectra, but this has been studied the most quantitatively for radio-loud quasars. The ratio of core to extended radio luminosity $R=L_{\text {core }} / L_{e x t}$ is frequently used as an orientation measure, on the assumption that $L_{\text {ext }}$ is emitted isotropically while $L_{\text {core }}$ is relativistically beamed. The forbidden lines from the NLR are assumed to be emitted isotropically. Wills \& Browne (1986) and Jackson \& Browne (1991) found that $\mathrm{FWHM}(\mathrm{H} \beta)$ and $W_{\lambda}([\mathrm{O} I \mathrm{II}])$ are inversely correlated with $R$ while $W_{\lambda}(\mathrm{H} \beta) / W_{\lambda}([\mathrm{OIII}])$ and $W_{\lambda}(\mathrm{Fe} \mathrm{II}) / W_{\lambda}([\mathrm{OIII}])$ are positively correlated with $R$. This was interpreted to mean that the quantities $L_{\text {core }}, L_{\text {opt }}, I(\mathrm{H} \beta)$ and $I$ (FeII) are all anisotropic to varying degrees. The consistent picture was that low-ionization lines like $\mathrm{H} \beta$ and Fe II were emitted from the surface of a rotating accretion disk, and looked brighter when the disk is viewed face on.

Several recent developments seem to be consistent with this model, or to add only digestible complications to it. Small values of $R$ (looking perpendicular to the radio jet) appear to correlate with increased reddening and increasing numbers of associated absorbers (Baker \& Hunstead 1995; Aldcroft et al. 1994; Wills et al. 1995). $W_{\lambda}([\mathrm{OII}])$ is found to anticorrelate more strongly with $R$ than does $W_{\lambda}\left(\left[\mathrm{O}_{\mathrm{III}}\right]\right)$, implying that $[\mathrm{O} \mathrm{III}]$ is also emitted somewhat anisotropically (Hes et al. 1993; Baker \& Hunstead 1995). A more detailed look at the behavior of $\mathrm{H} \beta$ shows that only the high-velocity gas in the line wings emits anisotrop- 
ically; the strength of the line center appears not to correlate with orientation (Brotherton 1996).

However, a new and surprising result (B. Wills 1997; D. Wills 1997) comes from the HST radio-loud sample, which includes $\sim 60$ QSOs chosen specifically to provide a wide range in the $R$ parameter in a carefully controlled sample. A principal-component analysis like the one done by Francis et al. (1992) indicates that the spectral variance which correlates with $R$ comes mostly from an overall change in the equivalent widths of all of the broad emission lines together, rather than anisotropic emission of just the low-ionization lines. This is a preliminary result which is still being examined.

\subsection{Radio-Loud vs. Radio-Quiet Objects}

A number of studies find significant differences between the BLRs of radio-loud and radio-quiet AGN. In interpreting the results, it is important to keep in mind that several underlying variables seem to drive many of the observed correlations. These include dependences on UV luminosity, on $\alpha_{o x}$ or $L_{x}$ (i.e. on the general shape of the ionizing continuum), on radio-loud vs. radio-quiet characteristics, and on orientation. It is not clear how many of these underlying drivers are really independent of each other, and in many cases there is much room for confusion about which of them has the primary correlation with other observed parameters.

To avoid these uncertainties it is necessary to compare samples which are matched as much as possible in the independent variables other than the one whose effects are being tested. One approach is to draw the program objects from samples which are complete to well-defined limits, so that it is known what has been left out. This is important if selection effects could alter the distribution of the variables being studied; emission-line equivalent widths are one type of variable where this is a concern. A second tack is just to find in the literature 'matched pairs' of objects which are the same in all primary variables except for the one which is to be varied in the test.

Boroson \& Green (1992) and Francis et al. (1993) used optically selected complete samples, and then found all of the radio sources in them. The only potential problem here for a radio-quiet vs. radio-loud comparison is the rather high radio frequency used, which might preferably pick out beamed radio sources. Corbin $(1991,1992)$ and Corbin \& Francis (1994) compared complete optically selected to complete radio selected samples, with careful attention to maintaining the same distribution of redshift and rest-UV luminosity in all samples. The one problem area here is the rather suspicious flux calibrations of some of the data used by Corbin (1991). The HST radio-loud sample (Wills et al. 1995) should also produce unbiased results; this is a complete sample down to a welldefined limit in extended radio flux, but supplemented by matched pairs which bring in additional core-dominated (flat radio spectrum) sources.

The next step down in reliability is to use samples for which the selection criteria are not well controlled, but for which at least the luminosity distributions seem to be comparable. The large number of QSOs studied for Palomar absorption-line work fall into this category, and are the basis for the work by Tytler \& Fan (1992), Wills et al. (1993) and Brotherton et al. (1994b). Brotherton (1996) has assembled another sample well-matched in redshift and luminos- 
ity. Comparisons of line profile properties and many line intensity ratios should still be reasonably unbiased in these papers.

However, samples drawing on the IUE or $H S T$ archives are subject to major selection effects, especially luminosity differences. For example, both Marziani et al. (1996) and Corbin \& Boroson (1996) have obtained ground-based spectra of $\mathrm{H} \beta$ of most of the objects for which UV spectra covering CIV are available in the HST archives. Corbin \& Boroson find that line asymmetry appears to correlate with continuum luminosity. However (as they make clear in their Fig. 5) the luminous objects in the archival data are mostly radio-loud while the less luminous ones are mostly radio-quiet, so it is possible that it is the radio properties which have the primary correlation with asymmetry (as has been found in other studies). The same luminosity differences between the radio-loud and radio-quiet objects pervade the Marziani et al. data. Non-simultaneous UV and optical spectroscopy is another potential problem when using these archives. Because of the wide wavelength coverage, these are interesting studies well worth doing, but their results need to be treated with caution.

Table 1 summarizes the best-established differences in the BLR spectra of different types of objects. References are given in separate columns for radio-

Table 1. Dependence of BLR Properties on Radio Properties.

\begin{tabular}{|c|c|c|c|c|c|c|}
\hline Parameter & $\begin{array}{l}\text { Radio } \\
\text { Quiet }\end{array}$ & $\begin{array}{l}\text { Radio } \\
\text { Loud }\end{array}$ & Refs. & $\begin{array}{l}\text { Steep } \\
\text { Radio }\end{array}$ & $\begin{array}{l}\text { Flat } \\
\text { Radio }\end{array}$ & Refs. \\
\hline $\begin{array}{l}\text { Equiv, Widths: } \\
\text { High Ioniz. Lines } \\
\text { Fe Il } \\
\text { [O III] }\end{array}$ & $\begin{array}{l}\text { Strong } \\
\text { Weak }\end{array}$ & $\begin{array}{l}\text { Stronger } \\
\text { Weak } \\
\text { Strong }\end{array}$ & $\begin{array}{c}1,2,3 \\
4 \\
4\end{array}$ & $\begin{array}{l}\text { Weak } \\
\text { Strong }\end{array}$ & $\begin{array}{l}\text { Strong } \\
\text { Weak }\end{array}$ & $\begin{array}{l}4,8 \\
4,8\end{array}$ \\
\hline $\begin{array}{l}\text { Shifts, Asymmetries: } \\
\text { CIV, CIII] } \\
\text { H } \beta \\
\text { High vs. Low Ioniz. }\end{array}$ & $\begin{array}{c}\text { Blue } \\
\text { Red or Blue } \\
\text { More } \\
\text { Different }\end{array}$ & $\begin{array}{c}\text { Red or Blue } \\
\text { Red } \\
\text { More } \\
\text { Alike }\end{array}$ & $\begin{array}{c}1,5,6,7 \\
4,6,7 \\
2,7,9,10\end{array}$ & More Red & Less Red & 7,8 \\
\hline
\end{tabular}

References: (1) Corbin \& Francis 1994; (2) Brotherton et al. 1994b; (3) Francis et al. 1993; (4) Boroson \& Green 1992; (5) Wills et al. 1995; (6) Sulentic et al. 1995; (7) Marziani et al. 1996; (8) Brotherton 1996; (9) Tytler \& Fan 1992; (10) Corbin 1990.

quiet vs. radio-loud differences and steep vs. flat radio source differences. Steep spectrum radio sources are generally extended, while flat spectrum sources are generally compact, so correlations with the radio spectrum shape are thought to measure orientation dependence.

An anticorrelation between the optical-Fe II and [O III] line strengths appears to be connected with the distinction between radio-loud and radio-quiet objects and also with the orientation-dependent steep vs. flat radio properties. Boroson \& Green (1992) found the Fe II/[O III] ratio to be the most strongly varying property in their analysis of the $\mathrm{H} \beta$ spectral region in BQS QSOs, and that it also correlates with asymmetries and widths of the broad emission lines. Corbin (1993) showed that this eigenvector also correlates with $L_{x}$. A few of the 
BQS QSOs in the Boroson \& Green study are radio-loud. The ones with steep radio spectra lie at the extreme weak end of the distribution of $W_{\lambda}(\mathrm{Fe}$ II), while the flat-spectrum sources scatter in the middle of the distribution. Given the connection to the radio spectrum shape, it is tempting to interpret these effects as being due to orientation, but Boroson \& Green suggested that the Fe II/[O III] results instead represent a competition for ionizing photons between gas in an optically thick torus and in the NLR.

Several studies find that the high- and low-ionization line profiles are more alike in radio-quiet than in radio-loud objects. Francis et al. (1993), Brotherton et al. (1994b) and Corbin \& Francis (1994) interpreted this in terms of a stronger ILR component in radio-loud objects. Sulentic et al. (1995), using the same data set as Marziani et al. (1996), found velocity differences between the C IV and $\mathrm{H} \beta$ lines which show dramatically different behavior for radio-loud and radio-quiet AGN, with a large range in C IV velocity shifts for the radio-quiet objects. Corbin \& Boroson (1996), using a sample containing many of the same objects, did not find such an effect. Comparison of the CIV velocities listed in the two papers for objects which appear in both samples shows a much smaller range in Corbin \& Boroson's measurements. This is presumably due to the fact that Sulentic et al subtracted an ILR-like component from the CIV profiles before measuring the line parameters while Corbin \& Boroson measured the entire observed profiles. Although there may be a physical justification for subtracting off some sort of ILR component (if it comes from a physically separate region), it is often very hard to know how to measure it in lines like CIV, because there is no unblended ILR profile which can be fitted into the narrow peak of the C IV line. Figures 1 and 3 of Marziani et al. (1996) illustrate their approach.

There are conflicting results regarding correlations between asymmetries in high-ionization lines like $\mathrm{CIV}_{\mathrm{V}}$ and $\mathrm{C}_{\mathrm{III}}$ ) and flat or steep radio spectrum. Corbin (1991) and Corbin \& Francis (1994) find that these lines have redward asymmetries in steep radio spectrum objects and blueward asymmetries in flat radio spectrum objects. Both Corbin \& Francis and Boroson \& Green find that in general the radio quiet and flat radio spectrum objects are more similar to each other than either is to the steep radio spectrum objects. However, Wills et al. (1995) find for the HST radio-loud sample that the flat radio spectrum ob jects tend to have redward asymmetries in CIV, while steep spectrum objects have more symmetrical lines and radio quiet objects show blueward asymmetries.

\subsection{Conclusions}

This section has described observational results mostly for objects which are 2-3 orders of magnitude more luminous than the ones studied through reverberation techniques. At this bright end of the AGN luminosity function, some individual objects show complicated structure, probably including outflowing winds. More generally, there there is a clear tendency to find asymmetric, blueshifted line profiles which point towards large-scale gas flows, and line-width and intensity effects which are probably due to orientation differences. This would at least seem to narrow the range of viable models to those with well-ordered velocity fields.

Acknowledgments. I am grateful to the Institute of Astronomy, Cambridge, UK, for hosting me during a sabbatical leave during which this paper 
was written, and to STScI for providing funding through grant GO-06093.0194A. I wish to thank Bob Carswell, Andy Fabian, Paul Hewett, Martin Rees, and Chris Reynolds for many illuminating conversations, and Gary Ferland, Kirk Korista and Bev Wills for answering an almost endless barrage of e-mail questions (during which they shut off their computers only once).

\section{References}

Aldcroft, T. L., Bechtold, J., \& Elvis, M. 1994, ApJS, 93, 1.

Alexander, T., \& Netzer, H. 1994, MNRAS, 270, 781.

Avrett, E. H., \& Loeser, R. 1988, ApJ, 331, 211.

Baker, J. C., \& Hunstead, R. W. 1995, ApJ, 452, L95; Erratum ApJ, 461, L59. Baldwin, J. A. 1977, MNRAS, 178, 67P.

Baldwin, J.A., Ferland, G.J., Korista, K.T., Carswell, R.F., Hamann, F., Phillips, M.M., Verner, D., Wilkes, B.J., \& Williams, R. E. 1996, ApJ, 461,664 .

Baldwin, J., Ferland, G., Korista, K., \& Verner, D. 1995, ApJ, 455, L119.

Barvainis, R. 1987, ApJ, 320, 537.

Binette, L., Prieto, A., Szusziewicz, E., \& Zheng, W. 1989, ApJ, 343, 135.

Boroson, T. A., \& Green, R.F. 1992, ApJS, 80, 109.

Bottorff, M., Korista, K., Shlosman, I., \& Blandford, R. D. 1997, this volume.

Brotherton, M.S. 1996, ApJS, 102, 1.

Brotherton, M.S., Wills, B. J., Francis, P. J., \& Steidel, C.C. 1994a, ApJ, 430, 495.

Brotherton, M.S., Wills, B.J., Steidel, C.C., \& Sargent, W. L. W. 1994b, ApJ, $423,131$.

Carswell, R. F., et al. 1991, ApJ, 381, L5.

Cassidy, I., \& Raine, D. J. 1996, A\&A, 310, 49.

Chiang, J., \& Murray, N. 1996, preprint.

Clavel, J., Wamsteker, W., \& Glass, I. S. 1989, ApJ, 337, 236.

Collin-Souffrin, S. 1986, A\&A, 166, 115.

Collin-Souffrin, S., \& Dumont, A.-M. 1989, A\&A, 166, 13.

Collin-Souffrin, S., Dyson, J.E., McDowell, J. C., \& Perry, J. J. 1988, MNRAS, 232,539 .

Collin-Souffrin, S., Dumont, A.-M., \& Nazarova, L. 1997, this volume.

Corbin, M.R. 1990, ApJ, 357, 346.

Corbin, M. R. 1991, ApJ, 371, L51.

Corbin, M.R. 1992, ApJ, 391, 577.

Corbin, M. R. 1993, ApJ, 403, L9.

Corbin, M.R., \& Boroson, T. A. 1996, ApJS, in press.

Corbin, M. R. \& Francis, P. J. 1994, AJ, 108, 2016.

Davidsen, A. F., Hartig, G. F., \& Fastie, W. G. 1977, Nature, 269, 203. 
Davidson, K., \& Netzer, H. 1979, Rep. Prog. Phys., 51, 715.

Done, C., \& Krolik, J. H. 1996, ApJ, 463, 144.

Elvis, M., Wilkes, B. J., McDowell, J., Green, R.F., Bechtold, J., Willner, S. P., Oey, M.S., Polomski, E., \& Cutri, R. 1994, ApJS, 95, 1.

Emmering, R. T., Blandford, R. D., \& Shlosman, I. 1992, ApJ, 385, 460.

Espey, B.R., Carswell, R.F., Bailey, J.A., Smith, M.G., \& Ward, M.J. 1989, ApJ, 342, 666; Erratum ApJ, 354, 763.

Ferland, G. J. 1996, 'Hazy, a Brief Introduction to Cloudy', University of Kentucky Physics Department Internal Report.

Ferland, G.J., Baldwin, J.A., Korista, K.T., Hamann, F., Carswell, R.F., Phillips, M., Wilkes, B., \& Williams, R. E. 1996, ApJ, 461, 683.

Ferland, G.J., Peterson, B. M., Horne, K., Welsh, W.F., \& Nahar, S. N. 1992, ApJ, 387, 95.

Francis, P. J., Hewett, P. C., Foltz, C. B., \& Chaffee, F. H. 1992, ApJ, 398, 476.

Francis, P. J., Hooper, E. J., \& Impey, C. D. 1993, AJ, 106, 417.

Gaskell, C. M. 1982, ApJ, 263, 79.

Glass, I. S. 1992, MNRAS, 256, 238.

Goad, M. R., O'Brien, P. T., \& Gondhalekar, P. M. 1993, MNRAS, 263, 149.

Gondhalekar, P.M., Horne, K., \& Peterson, B. M., editors, 1994, Reverberation Mapping of the Broad-Line Region in Active Galactic Nuclei (San Francisco: Astronomical Society of the Pacific).

Hamann, F., \& Ferland, G. J. 1993, ApJ, 418, 11.

Hes, R., Barthel, P. D., \& Fosbury, R. A. E. 1993, Nature, 362, 326.

Jackson, N., \& Browne, I. W. A. 1991, MNRAS, 250, 422.

Königle, A., \& Kartje, J.F. 1994, ApJ, 434, 446.

Koratkar, A.P., Kinney, A.L., \& Bohlin, R. C. 1992, ApJ, 400, 435.

Korista, K., Baldwin, J., Ferland, G., \& Verner, D. 1996, ApJS, in press.

Korista, K. T., et al. 1995, ApJS, 97, 285.

Krolik, J.H., \& Kallman, T.R. 1988, ApJ, 324, 714.

Kwan, J., \& Krolik, J.H. 1981, ApJ, 250, 478.

Laor, A., Bahcall, J. N., Jannuzi, B.T., Schneider, D.P. \& Green, R.F. 1995, ApJS, 99, 1.

Marziani, P., Sulentic, J.W., Dultzin-Hacyan, D., Calvani, M., \& Moles, M. 1996, ApJS, 104, 37.

Mathews, W. G. 1986, ApJ, 305, 187.

Mathews, W. G. 1993, ApJ, 412, L17.

Mathews, W. G., \& Ferland, G. F. 1987, ApJ, 323, 456.

Mushotzky, R.F., Done, C., \& Pounds, K.A. 1993, ARA\&A, 31, 717.

Netzer, H. 1991, in Active Galactic Nuclei, ed. T.J.-L. Courvoisier \& M. Mayor, (Berlin: Springer-Verlag), p. 57.

Netzer, H., Brotherton, M.S., Wills, B.J., Han, M., Wills, D., Baldwin, J. A., Ferland, G. J., \& Browne, I. W. A. 1995, ApJ, 448, 27.

Netzer, H., \& Laor, A. 1993, ApJ, 404, L51. 
O'Brien, P. T., Goad M. R., \& Gondhalekar P. M. 1994, MNRAS, 268, 845.

O'Brien, P. T., Goad M. R., \& Gondhalekar P. M. 1995, MNRAS, 275, 1125.

Perry, J. J., \& Dyson, J. E. 1985, MNRAS, 212, 197.

Peterson, B. M. 1993, PASP, 105, 247.

Peterson, B. M. 1994, in Reverberation Mapping of the Broad-Line Region in Active Galactic Nuclei, ed. P. M. Gondhalekar, K. Horne, \& B. M. Peterson (San Francisco: Astronomical Society of the Pacific), p. 1.

Rees, M. J. 1987, MNRAS, 228, 47P.

Rees, M.J., Netzer, H., \& Ferland, G.J. 1989, ApJ, 347, 640.

Roos, N. 1992, ApJ, 385, 108. Giannuzzo, E., Kidger, M., Mannucci, F., Stanga, R. M., \& Wamsteker, W. 1993, å, 274, 174.

Sanders, D. B., et al. 1989, ApJ, 347, 29.

Scoville, N., \& Norman, C. 1988, ApJ, 332, 163.

Shields, J. C., Ferland, G. J., \& Peterson, B. M. 1995, ApJ, 441, 507.

Smith, M.G., Carswell, R.F., Whelan, J. A. J., Wilkes, B. J., Boksenberg, A., Clowes, R. G., Savage, A., Cannon, R.D., \& Wall, J. V. 1981, MNRAS, $195,437$.

Sulentic, J.W., Marziani, P., Dultzin-Hacyan, D., Calvani, M., \& Moles, M. 1995, ApJ, 445, L85.

Tytler, D., \& Fan, X.-M. 1992, ApJS, 79, 1.

Verner, E.M., Ferland, G.J., Korista, K.T., \& Verner, D. A. 1995, BAAS, 27, 840.

Wanders, I. \& Peterson, B. M. 1996, ApJ, 466, 174.

Wilkes, B. J. 1986, MNRAS, 218, 331.

Wilkes, B. J., \& Carswell, R. F. 1982, MNRAS, 201, 645.

Wills, B. J., et al. 1997, this volume.

Wills, B.J., Brotherton, M.S., Fang, D., Steidel, C.C., \& Sargent, W. L.W. 1993, ApJ, 415, 563.

Wills, B. J., \& Browne, I. W. A. 1986, ApJ, 302, 56.

Wills, B. J., Netzer, H., \& Wills, D. 1985, ApJ, 288, 94.

Wills, B. J., Thompson, K. L., Han, M., Netzer, H., Wills, D., Baldwin, J. A., Ferland, G. J., Browne, I. W. A., \& Brotherton, M.S. 1995, ApJ, 447, 139.

Wills, D., et al. 1997, this volume. 